\title{
Vibrotactile communication of information about consonants: Vowels mask consonants
}

\author{
BARRY G. GREEN, JAMES C. CRAIG, and DAVID B. PISONI \\ Indiana University, Bloomington, Indiana
}

\begin{abstract}
The perception of consonants in consonant-initial [consonant-vowel (CV)] syllables was measured using a vibrotactile spectral display. The display coded acoustic frequency and intensity on the $24 \times 6$ vibrotactile matrix of an Optacon transducer. In the first experiment, we found that for consonants spoken in the context of /_a/, subjects could correctly identify eight consonants only about $60 \%$ of the time. The second experiment revealed similarly poor performance when subjects tried to identify a set of CV syllables in which the consonants differed only in their places of articulation. The final experiment showed that place of articulation could be distinguished much more reliably when the vowel following the consonant was shortened, thereby reducing backward masking. Display modifications that might improve the tactile perception of consonants by reducing temporal masking are discussed.
\end{abstract}

The perception of consonants poses one of the most difficult problems for communicating speech vibrotactually. This difficulty is thought to arise because, compared with vowels, the acoustic information associated with consonants is generally contained in relatively brief, low-energy segments of the speech signal. It has been a persistent question whether or not the tactile sense possesses adequate temporal resolution to mediate these brief spectral events (Kirman, 1973; Richardson \& Frost, 1977).

Most of the evidence to date has been negative. Guelke and Huyssen (1959) failed to achieve adequate identification of consonants with a multiplechannel spectral display after having reported promising results with vowels. Pickett and Pickett (1963) had better success with a tactile vocoder, but only for some consonants, and only in a discrimination task. Their data showed a tendency for good discrimination among voiced and voiceless fricatives, and between fricatives and stops. More difficult were the distinctions among stops, nasals, and laterals, and between voiced and voiceless stops (e.g., when the data were corrected for guessing, /m-b/ was discriminated only $56 \%$ of the time, and /p-b/ only $22 \%$ ). More recently, Sparks, Kuhl, Edmonds, and Gray (1978) used a sophisticated electrotactile device to study tactile identification of consonant-vowel (CV) syllables and found that six stop consonants

This research was supported by a grant from the National Science Foundation, PFR 80-17178. The authors thank Roger P. Rhodes for his indispensable contributions as computer programmer and research technician, and also Diane Kewley-Port, both for her helpful comments and for providing many of the stimuli used in the experiments. The authors' mailing address is: Department of Psychology, Indiana University, Bloomington, Indiana 47405. and two nasals could be recognized tactually only about $50 \%$ of the time; place of articulation proved most difficult to discern ( $40 \%$ correct). Scilley (Note 1) has since provided the most promising results, demonstrating that a significant number of words can be identified tactually using a linear spectral display on the forearm, and that three-letter words differing only in initial consonant (e.g., pet, bet, met) could be recognized over $80 \%$ of the time. However, Scilley's stimulus sets, which contained only three words each, were smaller than the sets tested by Sparks et al.

Consistent with the earlier attempts, our laboratory has shown that information about vowels contained in CV syllables can be conveyed quite adequately using a two-dimensional (frequency $x$ intensity) vibrotactile display (Green, Craig, Wilson, Pisoni, \& Rhodes, in press). The present study extended this work to examine the problem of communicating information about consonants with the same display. The aim of the study was not only to establish how well subjects could identify consonants, but also to help understand some of the processes underlying tactile recognition of speech signals. Three experiments were conducted: The first examined a range of consonants in the context of a single vowel; the second focused on perception of stop consonants, which were found to be particularly difficult to identify tactually; and the third examined the problem of temporal (backward) masking in the recognition of stop consonants.

\section{EXPERIMENT 1}

This experiment assessed the effectiveness of a two-dimensional spectral display as a way of transmitting information about consonants. 


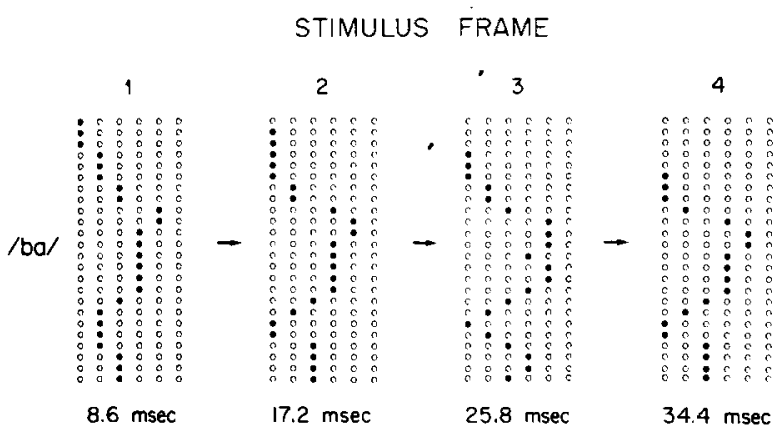

Figure 1. Pictured are the first four spectral frames of the stimulus $/ \mathrm{ba} /$. Filled dots represent the plas of the tactile display active during each 8.6-msec frame. Acoustic frequency is coded logarithmically along the 24 rows from $136 \mathrm{~Hz}$ (top row) to $3202 \mathrm{~Hz}$ (bottom row), and the energy in each of the 24 spectral channels is coded in 6-dB steps across the six columns of the display.

\section{Method}

The display consisted of the 144-pin, $24 \times 6$ vibrotactile matrix of an Optacon transducer (Hill, 1974; Linvill \& Bliss, 1966). The distribution of frequencies on the display was derived from the electrotactile spectral display of Sparks et al. (1978), which is a 36channel frequency-by-amplitude display with $1 / 3$-octave filter widths. Because the Optacon contains only 24 rows, the approximate center frequencies of only channels 10 through 23 (136 to $3203 \mathrm{~Hz}$ ) of the Sparks et al. display were adopted for the present display. The channels were distributed on the Optacon matrix such that the lower frequencies were represented near the top of the finger and the higher frequencies toward the bottom. The energy in each frequency channel was coded spatially across the 6 colums of the array in 6-dB steps. Hence, measurable energy in a given frequency channel was represented by the position of an active vibrator in the appropriate row of the Optacon. Information about the speech signal was updated every $8.6 \mathrm{msec}$ to create a real-time running spectral display that manifested the speech spectrum as a contour of vibration that changed shape over time. Figure 1 illustrates this tactile coding scheme by showing the first four spectral frames of the utterance $/ \mathrm{ba} /$.

The stimuli were single examples of eight consonants $(/ b, d, g$, $p, t, k, m, n /$ ) spoken once each in / $a /$ context by an adult male, originally in the carrier phrase "Teddy said (CV)." The carrier phrase was edited out, and the digitized signals were shaped into running spectra using Spectrum, a spectral analysis program based on linear prediction analyses (Kewley-Port, Note 2). The smoothed running spectra were then stored on disk and output to the Optacon via a PDP-11/34 computer equipped with appropriate interfaces. To remove duration as a potential cue to stimulus identity, all of the stimuli were truncated to 308.6 -msec frames (258 msec).

The stimuli were presented in random sequence to the index fingertip of the left hand, and the subjects responded by pressing one of eight numbered keys on a response terminal. A video monitor provided immediate feedback either by signaling that the response was correct or by displaying the number of the correct response. The subject initiated each trial with a keypress. A testing session contained 320 trials presented in eight blocks of 40 trials each, and subjects were told to take at least one brief break midway through the session.

Four normal-hearing, college-aged subjects served 9 days each to generate 2,880 trials per subject, 11,520 trials overall. The subjects had had experience (several thousand trials) in identifying vibrotactile representations of vowels, but they had never been asked to identify consonants in CV syllables.

\section{Results and Discussion}

The individual learning curves, averaged across consonants, are shown in Figure 2. A wide range of performances is evident across subjects, although over the last five sessions the standard error of the mean was only about $2 \%$. The average performance on the 9th day reached only $61.3 \%$ correct; one subject barely reached $40 \%$ correct, and the two best managed only $70 \%$ to $75 \%$ correct.

A post hoc analysis showed that manner of articulation was the feature communicated best by the display, with the subjects able to differentiate between the manner classes of stop and nasal $91 \%$ of the time. Voicing appeared less easily identifiable, with percentage correct reaching $81 \%$, and place of articulation was identified only $67 \%$ of the time. Performance was similarly poor when measured for the six stop consonants alone; the place feature was perceived correctly on only $64 \%$ of the trials in which stops were presented. The differences in performance among these speech features cannot be accepted as significant, however, because the features were represented by varying numbers of stimulus and response alternatives. Figure 3 demonstrates this point by showing the percentage of correct identifications for each feature along with the levels of performance that would be expected if the subjects merely had guessed on every trial. For example, even though manner of articulation was correctly identified $91 \%$ of the time, the subjects would be expected to score $62 \%$ correct by chance alone. ${ }^{1}$

Another experiment in which the feature classes are represented equally among the stimuli would be required to establish the saliency of the features with

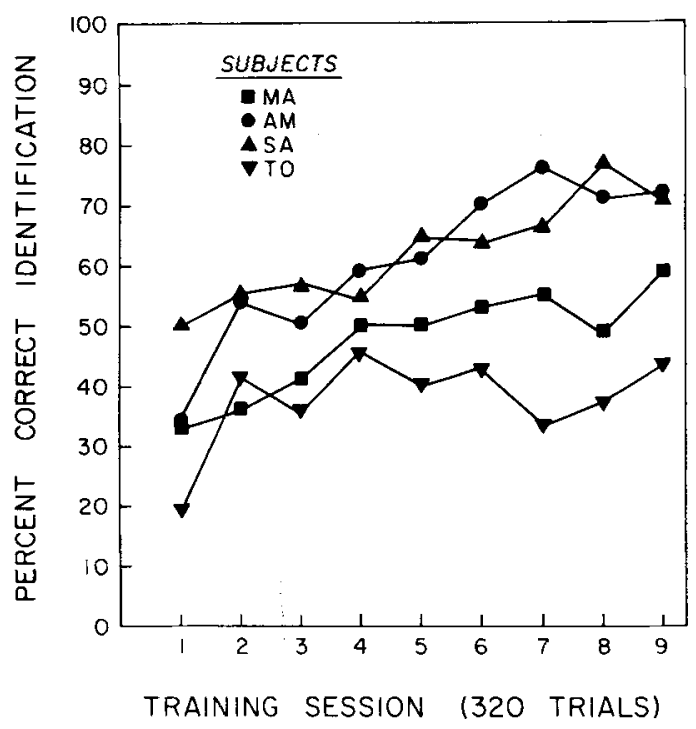

Figure 2. Percentage correct Identifications of eight CV syllables as a function of training session for four subjects. 


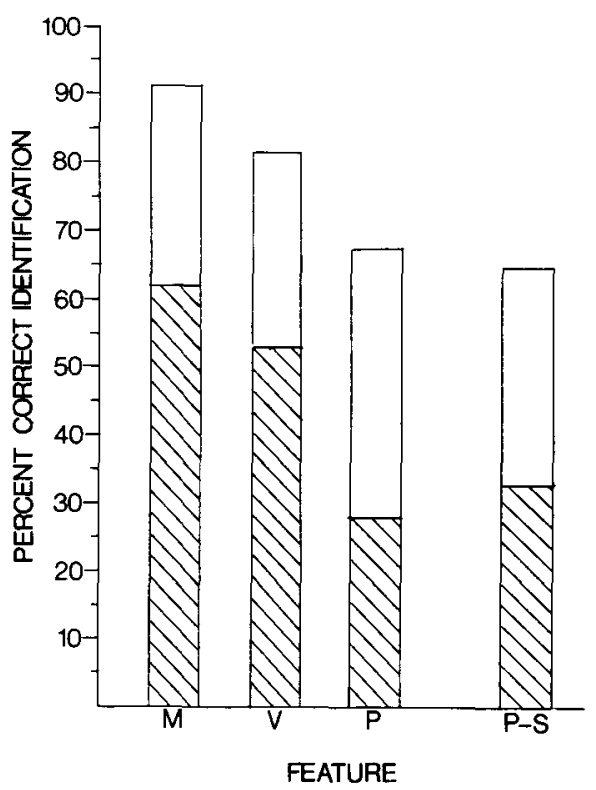

Figure 3. The consonant identification data of Figure 2 are shown broken down according to performance on specific consonantal features: $\mathbf{M}=$ manner of articulation, $\mathbf{V}=$ voicing, $\mathbf{P}=$ place of articulation, and $\mathbf{P}-\mathrm{S}=$ place of articulation for stop consonants only. The hatched areas denote chance performance. (Performance on the features is higher than on the syllables themselves becanse on some trials the subjects responded with the correct feature class but with the incorrect consonant.)

greater certainty. The present data are, however, similar to results for syllables presented aurally in noise (Miller \& Nicely, 1955), which suggests that in its present form the tactile display is incapable of communicating many of the subtle features of the speech signal.

From the standpoint of the utility of a tactile display, the results may not be quite as discouraging as the low percentages seem to imply. In speech-reading, the majority of errors result from an inability to perceive the presence or absence of voicing (Erber \& DeFilippo, 1978), a feature that seemed to be transmitted reasonably well both by the present spectral display and by the 36-channel electrotactile aid of Sparks et al. (1978). In addition, place of articulation, which seems poorly transmitted tactually, is a relatively strong speech-reading feature, whereas manner of articulation, which seems to be well communicated by the tactile display, is poorly perceived visually (Erber, 1972; Erber \& DiFilippo, 1978). Nevertheless, the low overall level of performance in the present experiment makes it clear that the display must be improved if speech perception is to be mediated primarily by the skin.

The problem becomes more complex when one considers that the present experiment employed only single tokens of consonants spoken in the context of the same vowel. Both of these factors might have contributed to artificially high consonant-recognition scores, the former by eliminating interstimulus variance owing to repeated utterances, and the latter by relieving the subject of the task of abstracting invariant consonantal cues from a variety of vowel contexts. In addition, cues unrelated to articulation of the consonant may have been available because of subtle differences in the vowel portion of the CVs (i.e., no two /a/s are precisely alike).

The problem of spurious vocalic cues to consonant identity was addressed in a follow-up experiment. The preferred way to attack the problem would have been to avoid the use of single tokens and instead to require subjects to identify a large number of tokens of each syllable. Because we were unable to convert "live voice" to vibrotactile patterns, that approach was impossible. An alternative strategy was used: A "standard vowel" was selected (from the stimulus $/ \mathrm{ba} /$ ) to replace most of the vocalic portion of the syllables, with the assumption being that if subjects were identifying the syllables by attending to vocalic cues, their performance should worsen when the differences among the vowels were reduced. The modified stimuli were created by eliminating the last 18 frames $(154.8 \mathrm{msec})$ of each CV syllable and replacing them with the last 18 frames of the stimulus /ba/. The resulting eight stimuli had spectra that differed only during their first $103.2 \mathrm{msec}$, which was more than adequate time to transmit information about stop consonants (Blumstein \& Stevens, 1979; Halle et al., 1957; LaRiviere, Winitz, \& Herriman, 1975; Kewley-Port, Note 2). This procedure did, however, leave some of the original vocalic information intact. This was necessary primarily because the nasal consonants, which are characterized by relatively slow formant transitions, would have suffered if more of the original syllable had been replaced. A frame-byframe comparison of $/ \mathrm{ma} /$ and $/ \mathrm{na} /$ showed that by the 12th frame $(103.2 \mathrm{msec})$ there were no significant spectral differences between them. ${ }^{2}$

The same subjects were tested for 10 sessions with the modified stimuli, and the individual data are plotted in Figure 4. A comparison with Figure 2 indicates that overall performance was inferior to what had been obtained with the original stimuli, and that the change to a standardized vowel seemed to hurt the two better subjects more than it hurt the two poorer subjects. It appears that the superior performance attained by the better subjects in the main experiment was owing in part to recognition of subtle differences in the vocalic portions of the syllables. Percentage correct for all four subjects averaged over the last five sessions was $\mathbf{9 . 5 \%}$ lower (48.3\%) for the prototypic stimuli than for the original stimuli $(57.8 \%)$. It is interesting, however, that confusions were almost identical for both stimulus sets: The stimulus that was most confused with each $\mathrm{CV}$ was the same in both conditions for seven of the eight CVs. The Spearman correlation between stimulus ranks 


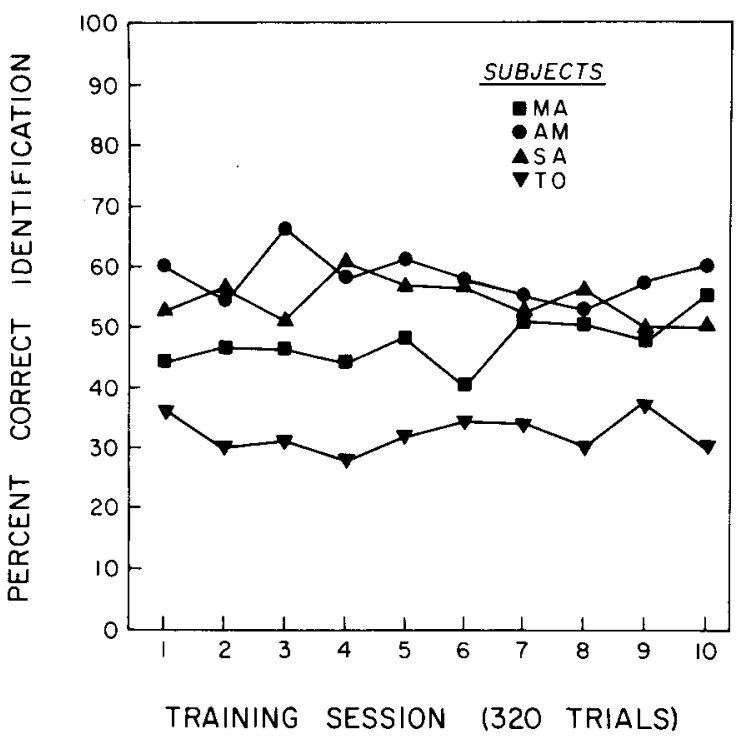

Figure 4. Same as Figure 2, except the CV syliables share identical vowel segments.

(when the stimuli were ordered from easiest to most difficult to identify) was .86 ( $p<.02)$.

Thus, we can conclude that the use of single examples of each syllable was misleading with respect to the absolute level of performance, but that, in both conditions, confusions arose primarily from differences among the consonantal portions of the stimuli. This is an encouraging result because it indicates that spectral information about consonants is a weak, but perceptible, feature of the present spectral display. The obvious question is: How can the saliency of that information be improved in order to raise performance to more useful levels? To begin to answer this question requires that we first learn the reasons for the difficulty in recognizing the consonantal features. The next two experiments examined the problem by focusing on the ability of subjects to identify the place of articulation of stop consonants, first with CV syllables of normal duration and later with CV syllables reduced in duration by truncating the spectral information associated with the vowel in each syllable.

\section{EXPERIMENT 2}

The difficulty subjects had in identifying place of articulation in Experiment 1 supported the previous findings and conclusions of other researchers that the tactile sense cannot adequately process rapidly changing, low-energy stimulus events. Although debate continues over exactly what combination of acoustic properties comprises the invariant cues to place, it is likely that the necessary information is contained in both the spectral and temporal aspects of the speech spectrum as it evolves from stop release to vocalic resonance in as little as 10 to $40 \mathrm{msec}$ (Blumstein \& Stevens, 1980; Pisoni, 1978; Stevens \& Blumstein, 1978; Kewley-Port, Note 2). Sparks et al. (1978) placed primary blame for poor tactile identification of stops on the brevity of the release bursts, implying that stop consonants occur too rapidly for the skin to perceive them.

Although the results of Sparks et al. (1978) together with those of Experiment 1 make it clear that tactile perception of stop consonants is indeed poor, the data nevertheless indicate that some information about the place feature can be transmitted tactually. The present experiment laid the groundwork for a more detailed investigation of vibrotactile perception of stops by requiring subjects to identify three different stop consonants spoken in the context of five different vowels. Because the acoustic characteristics of stop consonants vary with the vowel environment or context that precedes or follows them, it is important to assess consonant identification in different vowel environments. Bilabial stop consonants, for example, begin with an occlusion of the lips, followed by release; but the shape of the vocal tract (including the lips) depends upon the vowel that follows the release, and the spectra of the release burst and formant transitions reflect that shape (Fant, 1973). The present experiment examined the ability of subjects to learn to identify stop consonants when the spectral and temporal cues related to them vary with vowel context.

\section{Method}

The stimuli were 30 -frame $\mathrm{CV}$ syllables constructed as before: three voiced stop consonants, $/ b, d, g /$, spoken once each in the context of five vowels, $/ \mathrm{i}, \mathrm{e}, \mathrm{a}, \mathrm{o}, \mathrm{u} /$, by one adult male. This produced a set of 15 syllables, 5 each in the place categories of labial $(/ \mathrm{b} /)$, alveolar $(/ \mathrm{d} /)$, and velar $(/ \mathrm{g} /)$. The stimuli were identified only by the numbers 1 through 15 and were organized on the response keyboard in three rows of five with the $/ \mathrm{b} / \mathrm{s}$ in row 1 , $/ \mathrm{d} / \mathrm{s}$ in row 2 , and $/ \mathrm{g} / \mathrm{s}$ in row 3 . The two subjects who served in the experiment were chosen because they had had extensive experience in learning to identify vowels vibrotactually, but had never identified consonants. Testing continued for 15 sessions to produce 4,800 trials per subject (approximately 320 presentations of each stimulus).

\section{Results and Discussion}

The averaged data for the two subjects are plotted in Figure 5. The syllables were disappointingly difficult to identify, and following a rise in percentage correct of only about $8 \%$ between Sessions 1 and 5 , the identification of syllables benefited surprisingly little from continued practice. Through the last five sessions, the average overall percentage correct was $46.6 \%$ (filled squares, Figure 5). When scored for identification of the five vowels alone, performance was much better, reaching $86.9 \%$. The consonant was correctly identified on $51.0 \%$ of the trials, which, although poor compared with the vowel-identification percentage, was nevertheless sig- 


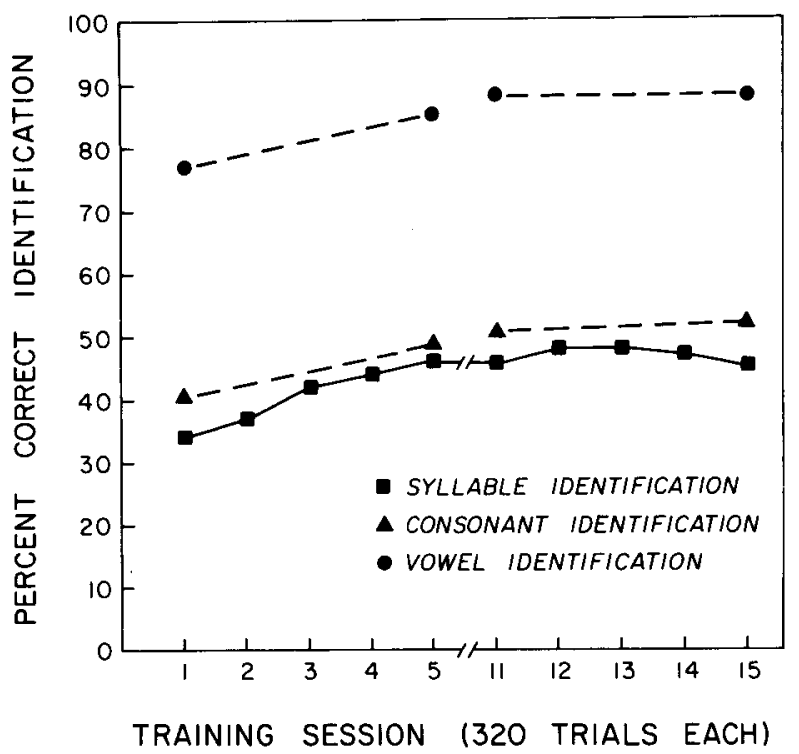

Figure 5. Percentage correct Identifications for $15 \mathrm{CV}$ syllables as a function of amount of practice. Note that the vowel in each syllable was more readily identified than the consonant (intermediate data points for vowels and consonants were omitted for simplicity and represented by dashed lines).

nificantly above chance performance $[t(4)=25.2$, $\mathrm{p}<.005] .^{3}$

There were no large differences in identifiability among the consonants. The alveolar / $d /$ was identified just slightly more frequently through the last five sessions than was the velar $/ \mathrm{g} /, 53.6 \%$ correct compared with $51.1 \%$, and the bilabial /b/ followed the others very closely at $\mathbf{4 8 . 3 \%}$ correct.

The pattern of confusions was consistent with what would be expected if identification of the vibrotactile representations of the consonants were based in part on perceived differences in the temporal onset of voicing: Confusions were more frequent between $/ b /$ and /d/ than between / $b /$ and $/ g /(34.9 \%$ vs. $23.9 \%$ ). Voicing onset time (VOT), the time between stop release and the beginning of voicing, increases as the locus of constriction of the consonant moves from the lips towards the velum (Lisker \& Abramson, 1964; Kewley-Port, Note 2), which might tend to make $/ \mathrm{b} /$ and $/ \mathrm{g} /$ more discriminable than $/ \mathrm{b} /$ and $/ \mathrm{d} /$ if VOT is a salient vibrotactile cue. Figure 6 illustrates how this temporal difference was manifested in the vibrotactile patterns for $/ \mathrm{bi} /, / \mathrm{di} /$, and $/ \mathrm{gi} /$. Notice that for $/ \mathrm{bi} /$,energy appears in the region of both the first and second formants (the two peaks in the spectrum) in Frame 1, and little change in the spectrum occurs beyond Frame 2 (after $17.2 \mathrm{msec}$ ). In comparison, /di/ begins with no significant energy at the location of the first formant (F1), and none appears there until $25.8 \mathrm{msec}$ after the release burst (Frame 3). Likewise, energy first appears at F1 for / gi/ after $25.8 \mathrm{msec}$, but becomes evident as a distinct waveform only in Frame 4, $34.4 \mathrm{msec}$ into the utterance.

The energy present above about $1000 \mathrm{~Hz}$ (nine rows from the bottom of the display) in the early frames of /di/ and /gi/ represents the acoustic energy in the release bursts. The spectral character of these bursts is also a possible cue to identification of stop consonants (Blumstein \& Stevens, 1979, 1980; Cole \& Scott, 1974; Halle, Hughes, \& Radley, 1957; Kewley-Port, Note 2). For the present stimuli, the burst might be expected to be more helpful in identifying $/ \mathrm{d} /$ and $/ \mathrm{g} /$ than in identifying $/ \mathrm{b} /$, because as Figure 6 shows, the shorter VOT for $/ b /$ reduces the distinctiveness of the burst. (Of course, the absence of a distinct burst may itself serve as a cue for $/ b /$.) With respect to $/ \mathrm{d} /$ and $/ \mathrm{g} /$, the frequency of the burst tends to be slightly higher for /d/ than it is for $/ \mathrm{g} /$, regardless of vowel context. The maximum in the aperiodic burst spectrum as measured in the first spectral frame occurs, on the average, about $1000 \mathrm{~Hz}$

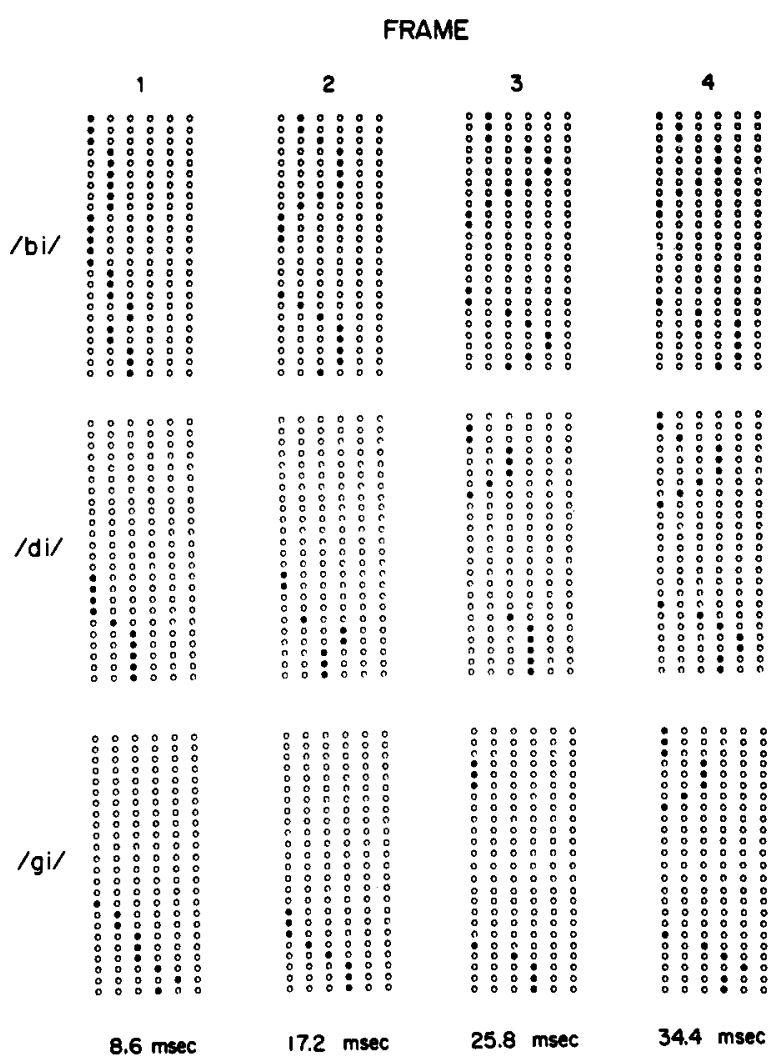

Figure 6. Shown are the first four framen of the vibrotuctle representations of the syllables / bl, d, g//. Active vibraton (filled dots) in the fint two frnmes of / $\mathrm{dV}$ and $/ \mathrm{g} / \mathrm{deplet}$ the release burst of the consonants; the appearance of activity near the top of the dinplay in the last two frames algnuls the onset of voicing (F1) for those stimall. Voicing may be present as early as the fint frame of $/ \mathrm{bL}$, makling the release bunt indheriminable as a separnte event. 
higher for /d/ than for $/ \mathrm{g} /(2968 \mathrm{~Hz}$ vs. $1933 \mathrm{~Hz})$. This trend is consistent with reports from studies that have examined the importance of the release burst in consonant recognition (Dorman, Studdert-Kennedy, \& Raphael, 1977; Halle, Hughes, \& Radley, 1957; Stevens \& Blumstein, 1978). However, much of the energy in the release burst occurs above the $3203-\mathrm{Hz}$ cutoff frequency of the present display; the burst may become a more viable cue if the cutoff frequency were raised to $4000-5000 \mathrm{~Hz}$, a change in the display that is being made.

Whatever the specific cues were that mediated identification of the consonants, they were considerably more difficult to extract from the stimulus displays than the cues used to identify vowels. The comparative ease with which the vowels were identified indicated that the difficulty did not lie in an inability to resolve and perceive steady-state spectral shapes, but rather in an inability to perceive rapid and brief spectral changes in the dynamic portion of the signal. The problem once again appears to be the inferior spatiotemporal resolution of the skin relative to the ear.

The resolution problem could take at least two forms: It might be related primarily (1) to the speed of the spectral changes associated with consonants or, alternatively, (2) to the relative brevity of the consonantal cues compared with the longer following vowel. In the first case, the tactile system might be so sluggish that tactile patterns associated with release bursts and formant transitions are blurred, therefore preventing distinctive information from being encoded. In the second case, the tactile system might be capable of processing the rapid spectral changes, but only if they occur in isolation. If the latter is true, temporal masking may be at the heart of the problem; the primary acoustic cues for stop consonants may be masked by the longer (and stronger) steady-state pattern of the following vowel. The next experiment was designed to test this possibility.

\section{EXPERIMENT 3}

The possibility that the vowel affects consonant identification in CV syllables was assessed by truncating the vocalic portion of the syllables. Because the consonant in CV syllables can be reliably identified aurally when as little as the first $20 \mathrm{msec}$ of a syllable is presented (Blumstein \& Stevens, 1980; Kewley-Port, Note 2), it was assumed that any improvements in performance that might result from shortening the 256-msec stimuli could be attributed to reductions in interference (masking) from the following vocalic portion of the syllable. No improvement would mean that the skin simply cannot process the rapid consonantal information.

\section{Method}

Three sets of 15 truncated syllables were created by eliminating the final 15, 20, or 25 frames of the 30-frame stimuli used in Experiment 2 . The resulting patterns had durations of 129,86 , and $43 \mathrm{msec}(15,10$, and 5 spectral frames), and were presented on the 24-channel display. The subjects' task was again to identify the 15 syllables, and the procedures were the same as before for initiating trials, recording responses, and receiving feedback.

Two of the three subjects who served in this experiment (hereafter referred to as Subjects 1 and 2) had participated in Experiment 1 . Those two subjects began by identifying the 15-frame stimuli, and subsequently received the 10 - and 5 -frame stimuli. The third subject, who had had no experience with the 30-frame stimuli, began by identifying the 5 -frame stimuli, and after having reached asymptote, was tested on the 30-frame stimuli. Subject 3 was added (after data collection was complete for the other two subjects) to control for possible order effects.

Experimental sessions were the same as before, containing eight blocks of $\mathbf{4 0}$ trials with feedback, and testing continued in each condition until performance approached asymptote.

\section{Results and Discussion}

Figure 7 displays the average results for the last five sessions in each condition for Subjects 1 and 2 (solid symbols). The most important finding was that perception of the consonants improved as the duration of the syllable decreased. This result means that the tactile system can indeed track at least some of the brief spectral cues associated with stop consonants, but that, in normal CV syllables, the tactile system fails to segregate the consonantal information from the subsequent vocalic information. Thus, consonant identification may be poor largely because of masking from the following vowel. However, even

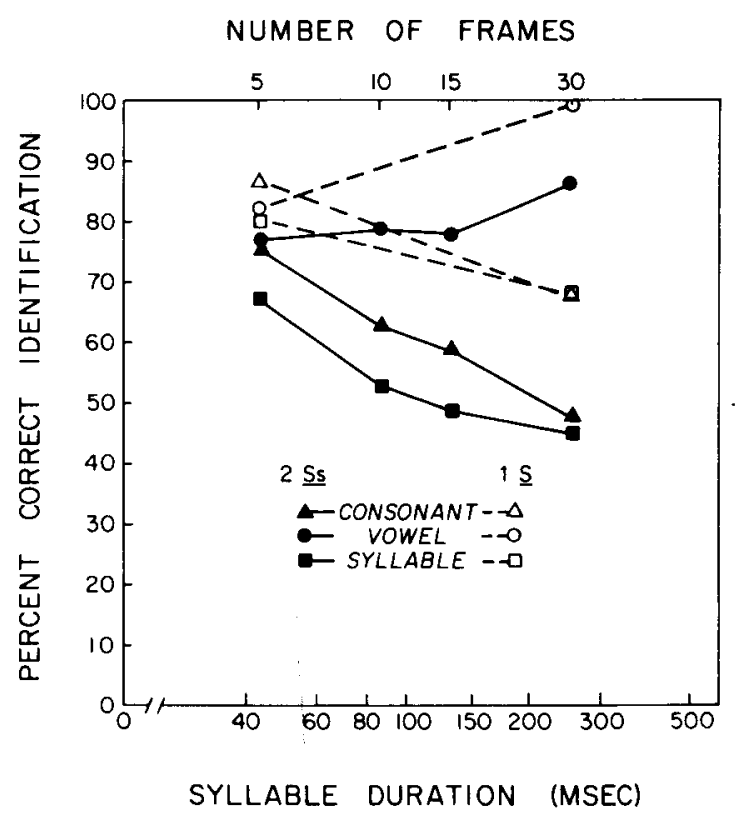

Figure 7. Percentage correct identifications as a function of syllable duration for $15 \mathrm{CVs}$. Dashed lines and open symbols show data for one subject who was tested only on the 5- and 30-frame stimuli. 
with masking reduced, performance was less than perfect, reaching at best only about $80 \%$ correct. This is probably because tactile processing of the rapidly evolving information in the first few frames is imperfect.

A linear regression analysis found that the relationship between consonant identification and syllable duration was best described by an inverse power function (if percent correct $=\mathrm{Y}$ and duration $=\mathrm{X}$, $\left.Y=148.3 \mathrm{X}^{-.2} ; \mathrm{r}=-.993\right)$. This indicates that the earlier spectral frames associated with the vowel interfere more with identification of the consonant than do the later frames (i.e., a given change in duration has a greater effect on masking at shorter vowel durations than at longer vowel durations). Craig (1978) showed that backward masking of vibrotactile patterns (letters of the alphabet) extends over at least $200 \mathrm{msec}$, and an analysis of some of Craig's unpublished data indicates that backward masking may also be an inverse power function of stimulus duration.

For Subjects 1 and 2, the total effect of reducing syllable duration from 258 to $43 \mathrm{msec}$ was to raise performance on the consonants by $24.6 \%$, from $51 \%$ to $75.6 \%$. The third subject, who had learned the five-frame stimuli first, confirmed the effect (open triangles and dashed lines, Figure 7). However, that subject produced a smaller advantage for the brief stimuli $(18.5 \%)$, probably because she did so well with the longer stimuli, exceeding the other subjects' performance by $20.3 \%$. It may be that experience with the truncated stimuli provided Subject 3 with cues that she subsequently managed to extract from the longer stimuli. Yet her performance was also superior with the five-frame stimuli $(11.5 \%$ better than that of the other subjects), which may indicate that she simply possessed better tactile acuity and was somehow less vulnerable to temporal masking.

Shortening the vocalic portion of the stimulus left the overall pattern of consonant confusions unchanged. Table 1 shows that whether the stimulus was 30 frames or 5 frames long, $/ \mathrm{b} /$ was most often confused with $/ d /, / d /$ with $/ g /$, and $/ g /$ with $/ d /$. This indicates that reducing information about the vowel left the cues to the consonants fundamentally unchanged; the "original" cues simply became more perceptible.

Table 1

Confusion Matrix for Consonants for 30- and 5-Frame Stimuli

\begin{tabular}{|c|c|c|c|c|c|c|}
\hline \multirow{3}{*}{$\begin{array}{l}\text { Stim- } \\
\text { ulus }\end{array}$} & \multicolumn{6}{|c|}{ Response } \\
\hline & \multicolumn{2}{|c|}{$/ \mathrm{b} /$} & \multicolumn{2}{|c|}{$/ \mathrm{d} /$} & \multicolumn{2}{|c|}{$\mid \mathrm{g} /$} \\
\hline & 5 & 30 & 5 & 30 & 5 & 30 \\
\hline $\begin{array}{l}\mid \mathrm{b} / \\
/ \mathrm{d} / \\
\mid \mathrm{g} /\end{array}$ & $\begin{array}{r}88.1 \\
6.8 \\
3.8\end{array}$ & $\begin{array}{l}56.1 \\
17.4 \\
15.9\end{array}$ & $\begin{array}{r}8.9 \\
75.4 \\
13.4\end{array}$ & $\begin{array}{l}26.7 \\
58.8 \\
29.2\end{array}$ & $\begin{array}{r}3.0 \\
17.8 \\
82.8\end{array}$ & $\begin{array}{l}17.2 \\
23.8 \\
54.9\end{array}$ \\
\hline
\end{tabular}

Note-Entries are percentages of total responses to each consonant.
Changing syllable duration affected vowel identification in an opposite and less dramatic way. Discarding 25 spectral frames reduced identification of the vowels by only $10.1 \%$ for Subjects 1 and 2 , and $16.2 \%$ for Subject 3. This comes to an average loss in vowel identification of $12.1 \%$, compared with an average gain in consonant identification of $21.6 \%$.

Because the stimuli used in the present experiment were originally derived from speech signals that were used in auditory experiments by Kewley-Port (Note 2), we have a unique opportunity to compare the vibrotactile results with results obtained during normal listening. As would be expected, listening to truncated CVs produced generally better identification scores than did feeling them (auditory identification was often perfect at syllable durations of $50 \mathrm{msec}$ or longer). However, some interesting comparisons can be made. For example, with respect to consonants, whereas $/ \mathrm{g} /$ was most difficult to identify aurally in Kewley-Port's experiment, /d/ was most difficult tactually in the present experiment. In the tactile task, performance on $/ \mathrm{d} /$ suffered because of confusions with both $/ \mathrm{b} /$ and $/ \mathrm{g} /$, whereas $/ \mathrm{g} /$ was usually confused only with $/ d /$. Part of the difficulty with / $d /$ might be attributable to the relatively low cutoff frequency of the present tactile display, which, as was pointed out above, fails to transmit the spectral information in the burst above about $3000 \mathrm{~Hz}$. Alternatively, this result might be expected because of the relatively coarse temporal acuity of the tactile sense. Whereas the difference in VOT between $/ b /$ and $/ g /$ may be large enough to be perceived rather easily tactually, the smaller differences between $/ \mathrm{b} /$ and $/ \mathrm{d} /$ and between $/ \mathrm{d} /$ and $/ \mathrm{g} / \mathrm{might}$ be less reliably perceived. The superior temporal acuity of the ear should reduce the number of such bipolar confusions between $/ d /$ and its two neighbors. Why /g/ was so difficult aurally is unclear: Although it is true that $/ g$ / requires a longer duration to complete its formant transitions, the principle difficulty was encountered with front vowels, with which, at durations comparable to those of the present experiment, $/ \mathrm{g} /$ was identified in the context of /i/ only about $60 \%$ of the time (Kewley-Port, Note 2). The subjects did better tactually, identifying $/ \mathrm{g} /$ in the same truncated syllable $\mathbf{7 8 . 8 \%}$ of the time.

A larger difference between the auditory and tactile data appeared for vowels: Aurally, KewleyPort (Note 2) found that identification of truncated vowels was consistently poorer than identification of consonants in the same syllables. In contrast, tactual performance tended to remain superior for vowels even at brief durations. In the auditory case, we would not expect truncation to improve the nearly perfect identification of consonants obtainable with syllables of normal length. However, truncation might disturb the auditory perception of vowels, because elimination of spectral information could change their phonetic quality. The shortened version 
of the vowel $/ 0 /$, for example, was particularly difficult aurally (identified only about $50 \%$ of the time at durations of $20-40 \mathrm{msec}$ ), because the truncated vowel no longer contains the glissando (falling F1 and F2) that provides much of its phonetic character. Perhaps if subjects received extensive auditory practice with the brief syllables, they would eventually learn to use subtle tonal differences to identify the unusual vowel sounds. The tactual subjects, having no existing "phonetic" categories with which to identify the vibrotactile stimuli, were forced at the outset to seek such subtle spectral (i.e., spatial) differences in order to learn the stimulus set: hence, the surprising result that some truncated vibrotactile vowels were identified as well as or better than some truncated auditory vowels.

\section{GENERAL DISCUSSION}

The results of the present experiments are both encouraging and discouraging with respect to tactile speech perception. It is discouraging to conclude that a two-dimensional vibrotactile spectral display such as the one used here appears incapable of communicating "useful" information about many kinds of consonants. The poor performance in Experiment 2 (about $50 \%$ correct with only three consonants) is probably well below what would be necessary for tactile aids to be adequate conveyors of connected discourse. The encouraging news is that for the first time there is evidence that, provided temporal masking is reduced, the tactile system (or at least that part of it serving the fingertip) may be "fast enough" to extract significant amounts of information from the brief stimulus events associated with consonants (i.e., the release bursts and formant transitions).

It may be useful at this point to discuss what appear to be paradoxical findings: that the skin is sufficiently acute temporally to apprehend consonants, and yet is also sufficiently sluggish to lose the consonants in the wake of the following vowels. The answer to this paradox may lie in the characteristics of the two sensory processes of temporal masking and temporal integration (Craig, 1982). To begin with, temporal (backward) masking and temporal integration (of patterns) appear to act over different intervals of time, with vibrotactile backward masking extending over at least $150 \mathrm{msec}$ (Craig, 1978) and temporal integration of patterns extending over at most 25 to 50 msec (Craig, 1982). Also, integration seems to have constructive as well as destructive consequences, whereas backward masking seems to have only destructive consequences. Integration is potentially constructive because it tends to combine sequential sensory events into unified percepts. Such unitary percepts may be advantageous when the contiguous stimulus events that they comprise are correlated and when no loss of information results from superimposing one event upon another. The im- provement in consonant identification in Experiment 3 apparently reflects this type of constructive integration, with the spectral (i.e., spatial) changes in the first few stimulus frames combining over time to form recognizable tactile patterns. Temporal integration may be helpful, and perhaps even necessary, for tactile perception of speech; over brief intervals, adjacent segments of the speech signal are acoustically related, and so should probably be perceived unitarily. It is this type of integration that Kirman (1973) argued must occur if speech is to be transmitted tactually.

On the other hand, if the speech signal were composed of independent spectral events, the effect of integration would likely be destructive, with unrelated stimulus patterns being combined into confusing and perhaps meaningless amalgams. Actually when viewed over temporal intervals of $100 \mathrm{msec}$ or more, the speech signal is composed of independent acoustic events, and therein lies the problem with backward masking. It is over the longer time periods that backward masking has its effects, causing stimuli that are by themselves perceptible to be obliterated or confused by subsequent, only distantly related, stimuli. Thus, although it may be necessary for the acoustic correlates of $\mathrm{CV}$ coarticulations to be integrated temporally, it is destructive when the information in the steady-state vowel interacts with the earlier dynamic information.

If tactile speech-analyzing aids that use the acoustic stream are to be successful, ways must be found to reduce backward masking. One obvious solution is to separate in time the information about consonants from the information about vowels. This approach is impractical, however, because vowels and consonants coexist in a virtually continuous stream of acoustic events. An alternative might be to separate some of the spectral cues for vowels and consonants spatially rather than temporally. For example, some of the aperiodic energy associated with consonants (particularly fricatives) occupies higher frequencies than the periodic energy in the first two or three formants of the vowel. Frication generally contains energy at frequencies above $3000 \mathrm{~Hz}$ (Miller \& Nicely, 1955), as do some release bursts (see Halle, Hughes, \& Radley, 1957). If some of the aperiodic energy in bursts and frication can be applied to locations on the skin that are perceptually separate from the locations of the (lower) vocalic frequencies, masking might be reduced and perception of consonants may improve. A dual spectral display, in which the high and low speech frequencies are applied to separate fingers, is now being developed in our laboratory. Because masking between tactile patterns may be reduced when they are presented to different fingers (Craig, unpublished results), it is hoped the dual display will reduce backward masking between the tactile patterns associated with high and low speech frequencies, and thereby enhance the 
clarity of some of the temporal and spectral cues to consonants.

\section{REFERENCE NOTES}

1. Scilley, P. L., \& Frost, B. J. Evaluation of a vibrotactile auditory prosthetic device for word recognition (Research report), Kingston, Ont., Queen's University.

2. Kewley-Port, D. Representation of spectral change as cues to place of articulation in stop consonants (Research on Speech Perception, Tech. Rep. 3). Bloomington: Indiana University, Department of Psychology, 1980.

4. Syrdal, A. K. Perception of consonant place of articulation (Research Laboratory of Electronics). Unpublished manuscript, MIT, 0000 .

\section{REFERENCES}

Blumstein, S. E., \& Stevens, K. N. Acoustic invariance in speech production: Evidence from measurements of the spectral characteristics of stop consonants. Journal of the Acoustical Society of America, 1979, 66, 1001-1017.

Blumstein, S. E., \& Stevens, K. N. Perceptual invariance and onset spectra for stop consonants in different vowel environments. Journal of the Acoustical Society of America, 1980, 67, 648-662.

Cole, R. A., \& Scort, B. The phantom in the phoneme: Invariant cues for stop consonants. Perception \& Psychophysics, 1974, 15, 101-107.

CraIg, J. C. Vibrotactile pattern recognition and masking. In G. Gordon (Ed.), Active touch. Oxford: Pergamon Press, 1978.

Craig, J. C. Temporal integration of vibrotactile patterns. Perception \& Psychophysics, 1982, 32, 219-229.

Dorman, M. F., Studdert-Kennedy, M., \& Raphael, L. J. Stop-consonant recognition: Release bursts and formant transitions as functionally equivalent, context-dependent cues. Perception \& Psychophysics, 1977, 22, 109-122.

ERBER, N. P. Auditory, visual and auditory-visual recognition of consonants by children with normal and impaired hearing. Journal of Speech and Hearing Research, 1972, 15, 413-422.

Ereer, N. P., \& DeFilippo, C. L. Voice/mouth synthesis and tactual/visual perception of /pa,ba,ma/. Journal of the Acoustical Society of America, 1978, 64, 1015-1019.

FANT, G. Stops in CV-syllables. In G. Fant (Ed.), Speech sounds and features. Cambridge, Mass: MIT Press, 1973.

Green, B. G., Craig, J. C., Wilson, A. M., Pisoni, D. B., \& RHODEs, R. P. Vibrotactile identification of vowel spectra. Journal of the Acoustical Society of America, in press.

Guelke, R. W., \& HuYssen, R. M. Development of apparatus for the analysis of sound by the sense of touch. Journal of the Acoustical Society of America, 1959, 31, 799-809.

Halle, M., Huahes, G. W., \& Radley, J.-P. A. Acoustic properties of stop consonants. Journal of the Acoustical Society of America, 1957, 29, 107-116.

HILL, J. W. Limited field of view in reading letter shapes with the fingers. In F. A. Geldard (Ed.), Conferences on cutaneous communication systems and devices. Austin, Tex: Psychonomic Society, 1974.

KIrMaN, J. H. Tactile communication of speech: A review and an analysis. Psychological Bulletin, 1973, 80, 54-74.
LaRiviere, C., Winitz, H., \& Herriman, E. Vocalic transitions in the perception of voiceless initial stops. Journal of the Acoustical Society of A merica, 1975, 57, 470-475.

Linvill, J. G., \& Bliss, J. C. A direct translation reading aid for the blind. Proceedings of the IEEE, 1966, 54, 40-41

Lisken, L., \& Abramson, A. S. A cross-language study of voicing in initial stops: Acoustical measurements. Word, 1964, 20, 384-422.

Millen, G. A., \& Nicely, P. E. An analysis of perceptual confusions among some English consonants. Journal of the Acoustical Society of America, 1955, 27, 338-352.

Pickett, J. M., \& Picket, B. H. Communication of speech sounds by a tactual vocoder. Journal of Speech and Hearing Research, 1963, 6, 207-222.

Pisoni, D. B. Speech perception. In W. K. Estes (Ed.), Handbook of learning and cognitive processes (Vol. 6). Hillsdale, N.J: Erlbaum, 1978.

Richardson, B. L., \& Frost, B. J. Sensory substitution and the design of an artificial ear. Journal of Psychology, 1977, 96, 259-285.

Sparks, D. W., Kuhl, P. K., Edmonds, A. E., \& Gray, G. P. Investigating the MESA (Multipoint Electrotactile Speech Aid): The transmission of segmental features of speech. Journal of the Acoustical Society of America, 1978, 63, 246-257.

Stevens, K. N., \& Blumstein, S. E. Invariant cues for place of articulation in stop consonants. Journal of the Acoustical Society of America, 1978, 64, 1358-1368.

\section{NOTwS}

1. Chance performance for a consonant feature (e.g., manner of articulation) was estimated by first calculating for each consonant category (e.g., stops) the joint probability that both the stimulus and the response would fall in that category, and then summing across the joint probabilities for all of the consonant categories within the feature class. For example, for manner of articulation, chance performance equaled the joint probability that subjects would respond with one of the six stops [probability $(p)=6 / 8=.75$ ] when the stimulus was a stop (also occurring with $p=6 / 8=.75$ ) plus the joint probability that subjects would respond with one of the two nasals $(p=2 / 8=.25)$ when the stimulus was a nasal $(p=2 / 8=.25)$. This is so because, when guessing occurs, the stimulus and response are assumed to be independent, and the joint probabilities equal the products of the stimulus and response probabilities; therefore, overall chance performance equals $(.75)^{2}+(.25)^{2}=.62$, or $62 \%$.

2. What effect this procedure might have had on the auditory perception of the same syllables is not known. The modifications were made in the tactile stimuli, not in the original auditory stimuli.

3. Calculation of $t$ in this instance was contingent upon the assumption that the data collected in consecutive daily sessions were independent. Normally, the assumption of independence is tenuous for repeated-measures designs; however, the present data were considered independent only when performance neared or reached asymptote, which was generally the case during the final sessions of each experiment.

(Manuscript received July 30, 1982; revision accepted for publication January 24, 1983.) 